\title{
Time-Based Readout of a Silicon Photomultiplier (SiPM) for Time of Flight Positron Emission Tomography (TOF-PET)
}

\author{
F. Powolny, E. Auffray, Member, IEEE, S. E. Brunner, E. Garutti, M. Goettlich, H. Hillemanns, \\ P. Jarron, Member, IEEE, P. Lecoq, Member, IEEE, T. Meyer, H. C. Schultz-Coulon, W. Shen, and \\ M. C. S. Williams
}

\begin{abstract}
Time of flight (TOF) measurements in positron emission tomography (PET) are very challenging in terms of timing performance, and should ideally achieve less than 100 ps FWHM precision. We present a time-based differential technique to read out silicon photomultipliers (SiPMs) which has less than 20 ps FWHM electronic jitter. The novel readout is a fast front end circuit (NINO) based on a first stage differential current mode amplifier with $20 \Omega$ input resistance. Therefore the amplifier inputs are connected differentially to the SiPM's anode and cathode ports. The leading edge of the output signal provides the time information, while the trailing edge provides the energy information. Based on a Monte Carlo photon-generation model, HSPICE simulations were run with a $3 \times 3 \mathrm{~mm}^{2}$ SiPM-model, read out with a differential current amplifier. The results of these simulations are presented here and compared with experimental data obtained with a $3 \times 3 \times 15 \mathrm{~mm}^{3}$ LSO crystal coupled to a SiPM. The measured time coincidence precision and the limitations in the overall timing accuracy are interpreted using Monte Carlo/SPICE simulation, Poisson statistics, and geometric effects of the crystal.
\end{abstract}

\section{INTRODUCTION}

$\mathbf{T}$ HE time of flight technique applied in PET could make a major improvement in sensitivity over standard PET methods [1]. So far TOF-PET detector heads have been implemented using PMTs, and the next step would be the replacement of the PMT with a silicon photomultiplier [2]. SiPMs offer obvious advantages in terms of compactness and tolerance to magnetic field, with an energy resolution comparable to that obtainable with a PMT. However, so far the time precision of a PET channel built with a LSO crystal and SiPM looks inferior to a PMT-equipped channel. Our paper aims to discuss

Manuscript received June 09, 2010; revised October 26, 2010 and January 20, 2011; accepted February 17, 2011. Date of publication April 29, 2011; date of current version June 15, 2011.

F. Powolny, E. Auffray, H. Hillemanns, P. Jarron, P. Lecoq, and T. Meyer are with the European Organization for Nuclear Physics (CERN), 1211 Geneva 23, Switzerland (e-mail: francois.powolny@cern.ch).

S. E. Brunner is with the University of Technology Vienna, A1040 Vienna, Austria.

E. Garutti and M. Goettlich are with Deutsches Elektronen-Synchrotron DESY, Notkestrasse D-22607 Hamburg, Germany.

H. C. Schultz-Coulon and W. Shen are with the Kirchhoff Institute for Physics, University of Heidelberg, D-69120 Heidelberg, Germany.

M. C. S. Williams is with the University of Bologna, I-40126 Bologna, Italy. Color versions of one or more of the figures in this paper are available online at http://ieeexplore.ieee.org.

Digital Object Identifier 10.1109/TNS.2011.2119493 a fast front end electronics system that could be the building block of a readout architecture for a multichannel ASIC in a TOF-PET detector. It also tries to identify the parameters limiting the ultimate timing precision achievable in a SiPM-PET detector channel. In particular our work investigates the limits in timing precision imposed by the readout electronics, the photon emission and the light transport in the scintillating crystal, and by Poisson statistics. Monte Carlo simulation is also used to generate sequences of random photons impinging on the SiPM, whereas HSPICE is used to study the entire readout channel response. These, taken together, simulate the complete chain from the photon generation to the output of the differential amplifier-discriminator.

Up to now the SiPM readout was configured as a single-ended connection to the front end amplifier inputs [3], [4]. In this work, however, we propose a differential connection to the front end amplifier. This type of input connection to a SiPM has the advantage of superior rejection of ground- and supply-voltage noise, a key feature for a fast multichannel readout ASIC for TOF-PET applications where common mode noise can easily be transformed to electronic time jitter [13].

To evaluate this novel approach, we have used an ASIC already developed for the time of flight detector of the CERN ALICE experiment (NINO) [5]. This ultra-fast multichannel differential amplifier-discriminator works as a leading edge discriminator that also encodes, via time over threshold, the energy with the width of the digital output pulse. Connected to a time-based readout system [6]-[9], [19] it constitutes an extremely fast, compact and cost effective TOF-PET readout system, especially if the ASIC integrates the complete processing channel. Moreover, since this differential amplifier-discriminator, currently available in $0.25 \mu \mathrm{m}$ CMOS technology, could be designed in even deeper CMOS technology [10], a large number of channels [11] could also be accommodated in such an ASIC together with the time-to-digital-converters TDCs [12].

The next section describes the principle of the differential current mode amplifier-discriminator and its connection to the SiPM; thereafter we present the Monte Carlo simulation results of photon production in LSO, as well as the HSPICE circuit model of the SiPM together with simulation results of the entire channel. Section IV describes timing and calibration measurements with a laser. In Section V we describe the experimental setup used to determine the time precision, and in Section VI 


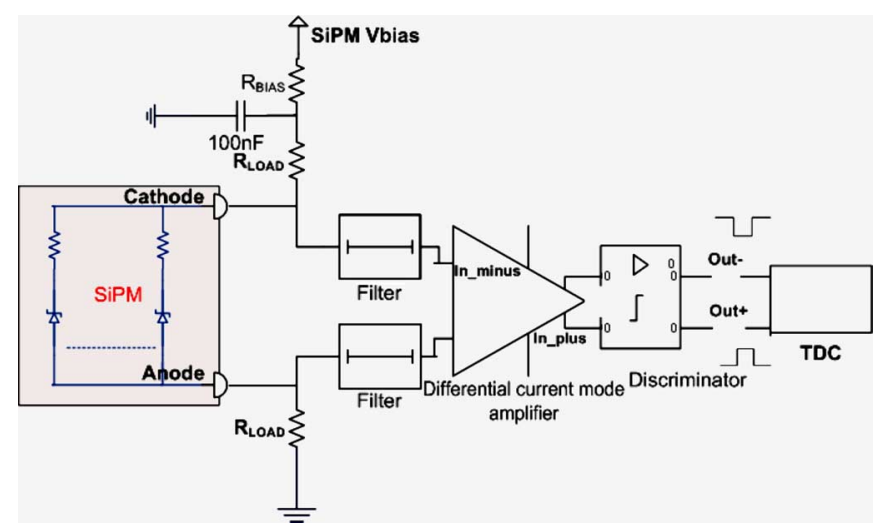

Fig. 1. Principle of the differential time-based readout. The circuit has a differential configuration from the SiPM terminals to the input of the TDC.

we present our experimental results and compare them with the simulations.

\section{FRONT-END AMPLIFIER DisCRIMINATOR (NINO)}

Several factors affect the timing performance of a PET detector: photon statistics, photon transport, the rise time and decay time of the crystal scintillation, the quantum efficiency of the photodetector and the avalanche timing uncertainty of the SiPM. In contrast to these, the timing limitations of the amplifier-discriminator circuit are far less severe, and therefore the amplifier-discriminator performance is more easily optimized for time jitter, being substantially lower than that produced by the scintillator and the SiPM. It is in fact feasible to set the discriminator threshold sufficiently low to fire on a single photon, thus minimizing the jitter provoked by photon statistics alone. Each readout channel of the SiPM is then made of two functionally different circuits (see Fig. 1): First, the SiPM's anode and cathode are both connected to the inputs of the differential current mode amplifier stage, which is in turn followed by a leading edge discriminator stage employing time-over-threshold processing. The digital output pulse with its leading edge provides the time stamp, which if necessary could be corrected for time walk via the output pulse width, whereas the pulse width on its own is used to encode the photon energy.

\section{A. Differential Time-Based Readout Technique}

The time-based readout system, encoding photon energy with the width of the discriminator pulse, also offers the advantage of filtering the acquired events for those contained in the photoelectric peak (e.g. 350-550 keV), i.e. directly at the output of the discriminator stage. This can be achieved with a simple digital circuit that vetoes the discriminator output for low energy photon signals (e.g. due to Compton scattering) and SiPM dark noise. This feature is particularly useful when the discriminator threshold is set to a value close to that of a single photon where the background is expected to be large.

Referring to Fig. 1 the differential connection between the SiPM and the amplifier inputs is obtained by adding a series load resistor to the cathode and anode terminals where the anode is referenced to ground. In this scheme the SiPM is connected

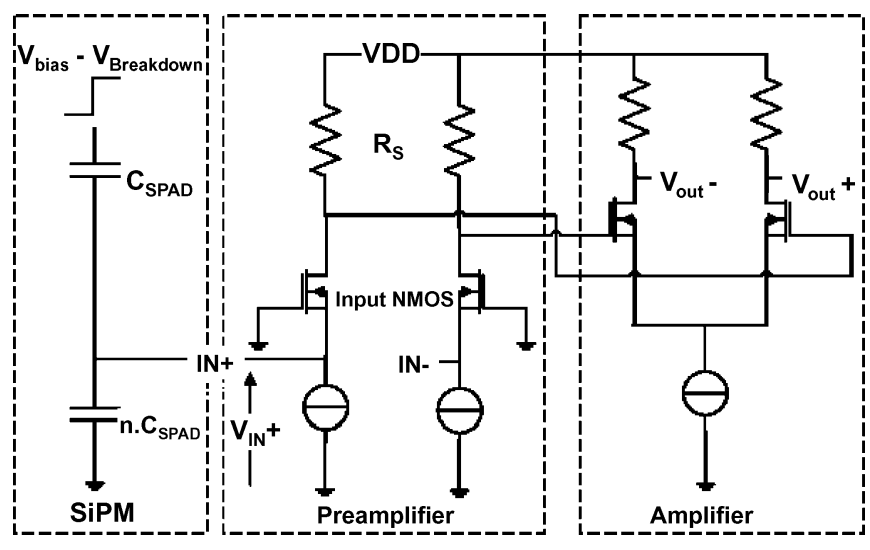

Fig. 2. Circuit principle of the differential amplifier input stage. For simplicity, cascode stages, additional current sources and DC stabilization are not drawn.

to the readout system based on a concept previously developed and successfully implemented in the TOF-MRPC detector of the ALICE Experiment having a timing precision of $\leq 60 \mathrm{ps}$ FWHM. The LVDS ${ }^{1}$ discriminator outputs are then connected to a TDC completing the time-based readout architecture [13].

\section{B. Optimization of the Input Stage Amplifier With the SiPM}

The preamplifier stage is optimized for single photon event sensitivity in order to detect the very beginning of a signal delivered by the LSO crystal. The optimization is done on the existing differential amplifier-discriminator ASIC (NINO), described in detail elsewhere [5]. It is based on a common gate input transistor pair with a differential configuration to sense the unbalanced current produced by the input signal. At the input the circuit can work with a single ended configuration or with a differential connection to the SiPM with the other negative input referred to ground. Fig. 1 shows the simplified equivalent circuit of the amplifier and SiPM for a single input circuit configuration to assess circuit optimization.

The simplified SiPM-amplifier circuit of Fig. 2, without the cascode stages and additional current sources, comprises the capacitance of a single $\mathrm{SPAD},{ }^{2} C_{S P A D}$, transferring the SPAD charge to the total capacitance of $\left(n \times C_{S P A D}\right)$ of the SiPM terminal when an avalanche occurs. $C_{S P A D}$ is the equivalent circuit of the junction capacitance, and $n$ is the number of SPAD cells of the SiPM; parasitic capacitances are neglected. Therefore the signal voltage at the input of the trans-conductance preamplifier triggered by a single SPAD avalanche is:

$$
V_{\text {in }} \approx\left(V_{\text {Bias }}-V_{\text {Breakdown }}\right) / n
$$

Taking $R_{S}$ as the drain resistor of the input transistor that senses the current unbalance in the two input branches the following signal is delivered to the second stage:

$$
\Delta V_{S} \approx g m s \times R_{S}\left(V_{\text {Bias }}-V_{\text {Breakdown }}\right) / n
$$

${ }^{1}$ Low Voltage Differential Signal.

${ }^{2}$ Single Photon Avalanche Diode. 
In case of the Hamamatsu SiPM with 3600 SPAD cells and a typical over-bias of $1.5 \mathrm{~V}$ (voltage above the Geiger-breakdown voltage), the input voltage signal of the preamplifier is about $400 \mu \mathrm{V}$ for a single photon (one fired pixel or SPAD). Sensing such a low signal on a capacitance as high as $320 \mathrm{pF}$, which is typical for $3 \times 3 \mathrm{~mm}^{2}$ devices like the Hamamatsu S10931-33-50C, requires a careful amplifier design. The input transistor should be dimensioned with large transconductance, since this maximizes the amplifier's sensitivity and, at the same time, minimizes series noise. The chosen source transconductance gms of $50 \mathrm{mS}$ lowers the amplifier input resistance to ground to $20 \Omega$, thought to be sufficient for an acceptable input signal time constant of the SiPM. The Equivalent Noise Charge ENC, with the SiPM connected to the amplifier, is about $5 \mathrm{fC}$ rms with an amplifier peaking time of $1.5 \mathrm{~ns}$. For a single pixel fired, the jitter would be about $100 \mathrm{ps}$ for a signal-to-noise ratio of 15 and a rise time of $1.5 \mathrm{~ns}$. In the case of a $511 \mathrm{keV}$ photo event the number of photoelectrons detected within the first ns is $\approx 30$ yielding, under a conservative assumption of a SiPM gain of $10^{5}$, a total electron charge of $3 \cdot 10^{6}$. With $5 \mathrm{fC}$ noise or $3 \cdot 10^{4}$ electrons rms the time jitter $\sigma_{e}=E N C /(d Q / d t)=$ $3 \cdot 10^{4} /\left(3 \cdot 10^{6} / 1 \cdot 10^{-9}\right)=10$ ps rms or $\approx 20$ ps FWHM.

The amplifier input stage is not linear, saturating for an input current of about $250 \mu \mathrm{A},{ }^{3}$ whereas at $511 \mathrm{keV}$ photon-energy the SiPM current reaches a peak value of $\approx 3 \mathrm{~mA}$. This feature enhances channel sensitivity at low discriminator thresholds by providing a larger gain at the very beginning of the SiPM signal. Hence the non-linearity of the input amplifier functions as a time-over-threshold signal processor before the discriminator stage sets in. To optimize the time-over-threshold response with a SiPM signal a passive differential filter has been inserted between the SiPM terminals and the amplifier inputs. This filter shapes the SiPM signal to extend the linear response of the time over threshold discriminator above the $511 \mathrm{keV}$ photo-peak signal amplitude.

\section{Simulation OF the TOF-PET ChanNEL}

The timing precision of a PET detector is dominated by the time spread due to the intrinsic photon emission process [14] and the photon transport in the scintillator. Another limitation comes from the scintillation rise time in the emission process which is estimated between $100 \mathrm{ps}$ to $500 \mathrm{ps}$ for a crystal made of LSO [27]. This latter effect has not been implemented yet in our simulation model. Our model so far includes all components of the detector channel, from the scintillation crystal (LSO) to the output of the discriminator. Photon emission statistics are dealt with Monte Carlo simulation on one hand, whereas the electronic effects in the chain, from the SiPM to the discriminator, are treated with HSPICE. As such, the output of the Monte Carlo simulation is fed as input into the HSPICE circuit model.

${ }^{3}$ It should be noted that the drain current of the input devices of the NINO discriminator is about $2 \mathrm{~mA}$, i.e. 10 times the signal saturation once the signal saturation of $250 \mu \mathrm{A}$ is reached. In signal saturation condition, the output nodes of the first stage are clamped limiting the voltage difference between the output branches to $400 \mathrm{mV}$. In these conditions the effect of the saturation is negligible and does not influence the response time of the discriminator.

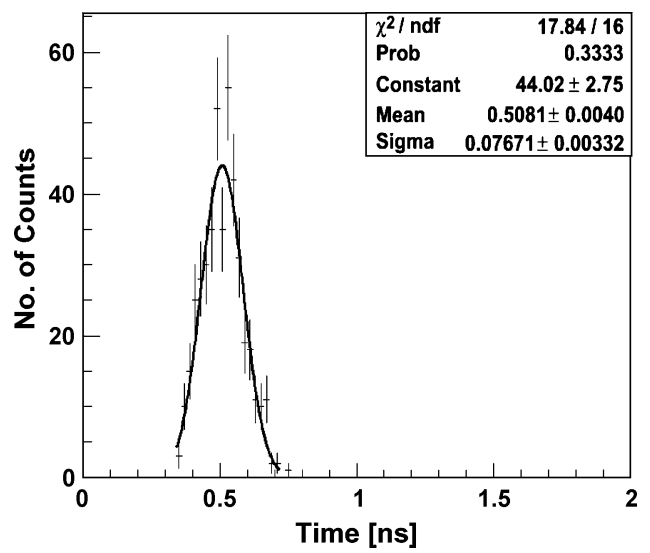

Fig. 3. Arrival time fluctuation of the 5th photon. The simulation includes photon statistics and the effects of photon transport in the LSO crystal.

TABLE I

Monte CARLo Simulation PARAMETERS

\begin{tabular}{cccc}
\hline $\begin{array}{c}\text { Light Yield } \\
\text { [photons / MeV] }\end{array}$ & $\begin{array}{c}\text { decay Time } \\
\text { [ns] }\end{array}$ & $\begin{array}{c}\text { Refractive } \\
\text { Index }\end{array}$ & $\begin{array}{c}\text { Absorption } \\
\text { Length }\left[\mathrm{cm}^{-1}\right]\end{array}$ \\
\hline 20,000 & 40 & 1.82 & 0.28 \\
\hline
\end{tabular}

\section{A. Monte Carlo Simulation of Light Production in LSO}

In Fig. 3 we show the results of the Monte Carlo simulation carried out with Geant 4 [15], [16] for a polished and fully wrapped (Teflon) LSO crystal of $3 \times 3 \times 15 \mathrm{~mm}^{3}$ oriented along the Line of Response (LOR) in a PET detector. It should be noted that at this time of our simulations no further investigations on crystal surface roughness have been made. The reflections in this crystal are specular. It shows the fluctuation in the arrival time of the 5th photon collected by the SiPM. The most important figures which limit the time resolution and which are taken into account here, are the light yield, the decay time and the spread in the depth of interaction of the incident $\gamma$-ray. The main simulation parameters are shown in Table I. Both, the wavelength dependent photon detection efficiency of the SiPM [17] and the LSO emission spectrum are taken as input to the simulations.

Note that the absorption length concerns the incoming gamma and not the scintillation photons. Under these assumptions the fluctuation in the 5th photon arrival time is $77 \mathrm{ps} \mathrm{rms}$, seen in a single detector, corresponding to a coincidence time precision of $\approx 250$ ps FWHM. Consequently, in the case of the 1st photon to be detected, not shown here, the coincidence timing fluctuation would improve to 122 ps FWHM. This sets a lower limit to the achievable time precision in a TOF-PET system, for it effectively is the irreducible, physical limit imposed by the LSO crystal of the tested size of $3 \times 3 \times 15 \mathrm{~mm}^{3}$ when exposed to $511 \mathrm{keV} \gamma$-rays. The result clearly shows that the ideal detection threshold should be at the level of one photon; however due to the SiPMs dark noise, this is rather difficult to achieve.

\section{B. Electronic (SPICE) Modeling of the SiPM}

The precise electrical modeling of the SiPM is an important ingredient in the understanding of the timing performance of 


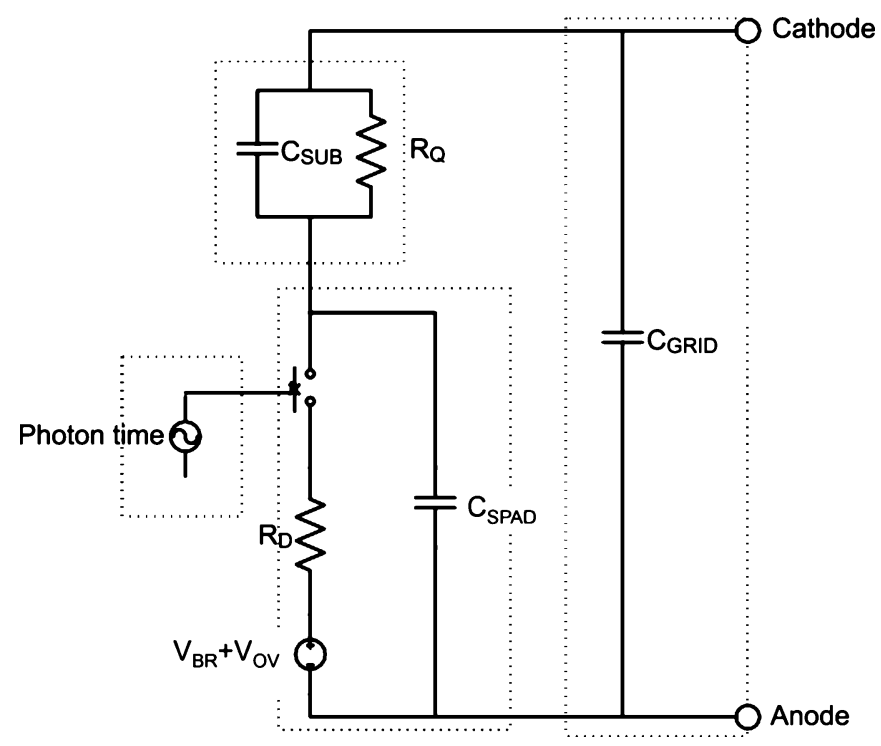

Fig. 4. SPICE model of the SPAD cell. Simulation includes 3600 cells connected in parallel. In this circuit, $C_{S P A D}$ is the effective SPAD capacitance, $R_{D}$ the junction series resistance, $R_{Q}$ the bias resistance, $C_{S U B}$ the substrate capacitance, $C_{G R I D}$ the interconnect capacitance, $V_{B R}$ the breakdown voltage, and $V_{O V}$ the over-bias voltage.

the complete PET detector chain. We have chosen to model the Hamamatsu SiPM S10362-33-050c with CADENCE HSPICE assuming an electrical circuit to be as close as possible to the physical device, based on the Cova model [18]. The equivalent circuit of a SPAD cell is shown in Fig. 4.

It comprises four functionally important blocks, i.e. the SPAD diode core, the quenching resistor, the avalanche trigger generator and the interconnect capacitance. The complete SiPMSPICE model then consists of an array of 3600 such SPAD circuits interconnected with each other in parallel.

To 'generate' an avalanche, a time dependent resistance acting as a switch, is used in each SPAD cell. In HSPICE the avalanche time is a sequential parameter that individually feeds each of the 3600 SPAD cells of the SiPM with the photon time sequence previously generated by the Monte Carlo simulation; this is explained in Section III-A. The relevant HSPICE parameters and their values used for the SiPM (Hamamatsu S10362-33-050c) are shown in Table II. Parameters have been estimated from the pulse shape of the single photon signal response. Note, from our comparison of the single-photon-SPICE results with the experimental results (see Section VI), some of the key parameters in the model, such as the experimental rise time, the signal amplitude and decay time, due to their uncertainties, had to be adjusted to achieve optimum conformity with the data. For example, we have observed that a series inductance (not shown) in the SPAD diode circuit of Fig. 4 significantly influences the timing behavior of the circuit to the extent that it may override the effect of the parameterized resistor $R_{D}$ in the model.

\section{Simulation Results of the Entire Readout Channel}

For a single photon event the unbalanced current signal at the differential input ports of the amplifier is $20 \mu \mathrm{A}$. Then the voltage signal across the $2.5 \mathrm{k} \Omega$ sensing resistors $R_{S}$ (see Fig. 2)
TABLE II

PARAMETERS OF THE HSPICE MODEL

\begin{tabular}{ccccccc}
\hline$R_{D}$ & $\begin{array}{c}V_{B R} \\
{[\Omega]}\end{array}$ & $\begin{array}{c}V_{O V} \\
{[\mathrm{~V}]}\end{array}$ & $\begin{array}{c}C_{S P A D} \\
{[\mathrm{~V}]}\end{array}$ & $\begin{array}{c}R_{Q} \\
{[\Omega]}\end{array}$ & $\begin{array}{c}C_{G R I D} \\
{[\mathrm{fF}]}\end{array}$ & $\begin{array}{c}C_{S U B} \\
{[\mathrm{fF}]}\end{array}$ \\
\hline $1 \mathrm{k}$ & 69.5 & 1.5 & 65.5 & $30 \mathrm{M}$ & 16 & 50 \\
\hline
\end{tabular}

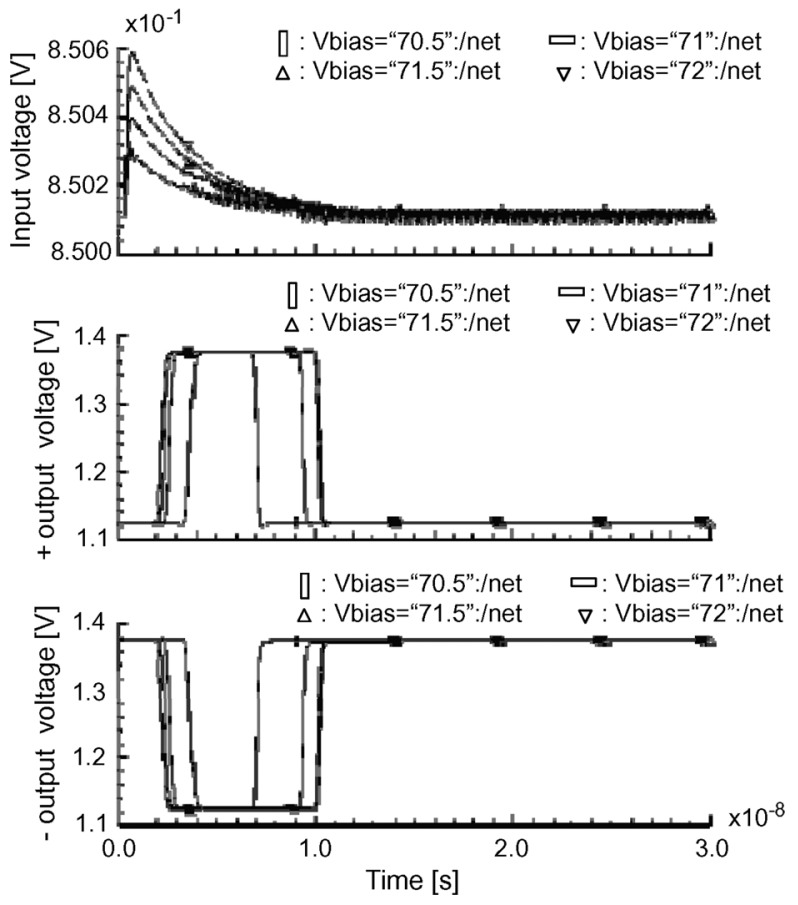

Fig. 5. Single photon HSPICE simulation of the discriminator with the SiPM connected for four over-bias voltages between $1 \mathrm{~V}$ and $2.5 \mathrm{~V}$. Amplifier input amplitudes (top graph) range from $200 \mu \mathrm{V}$ (@ $1 \mathrm{~V}$ over-bias) to $500 \mu \mathrm{V}(2.5 \mathrm{~V}$ over-bias). The corresponding pulse widths of the discriminator (bottom graphs) then vary between 3 ns and 8 ns.

is $50 \mathrm{mV}$, sufficient to detect a single photon. Fig. 5 shows the simulation results obtained for four different bias conditions and with the discriminator threshold set to the minimum, i.e. $50 \mathrm{mV}$.

When $511 \mathrm{keV} \gamma$-rays illuminate the LSO crystal typically 10,000 photons are impinging on the SiPM, over a duration, i.e. an exponential signal fall time, of $40 \mathrm{~ns}$. From the Monte Carlo simulation we know that the number of photoelectrons contributing to the SiPM signal is about 2000, taking into account the quantum efficiency of the SiPM and the optical characteristics of the crystal. Single photon signals then pile up on each other to form the signals shown in Fig. 6.

The corresponding discriminator pulse widths vary with the SiPM current signal, e.g. from 20 ns for an input signal equivalent to 100 photons to $120 \mathrm{~ns}$ for a 2000 -photon signal. As we had indicated in Section II-B and expected from the time-overthreshold stage, the discriminator response is nonlinear. However, this nonlinear response has the advantages that it encodes signals with a very large dynamic range, i.e. from 1 to 2000 photons, and that in a short time and via simple hardware processing, offering in addition the possibility of selecting events in the energy window around the photoelectric peak. This characteristic raises the important question on how many initial photons are needed for the amplifier-discriminator to operate at a stable discrimination level. For $511 \mathrm{keV}$, for example, the LSO 

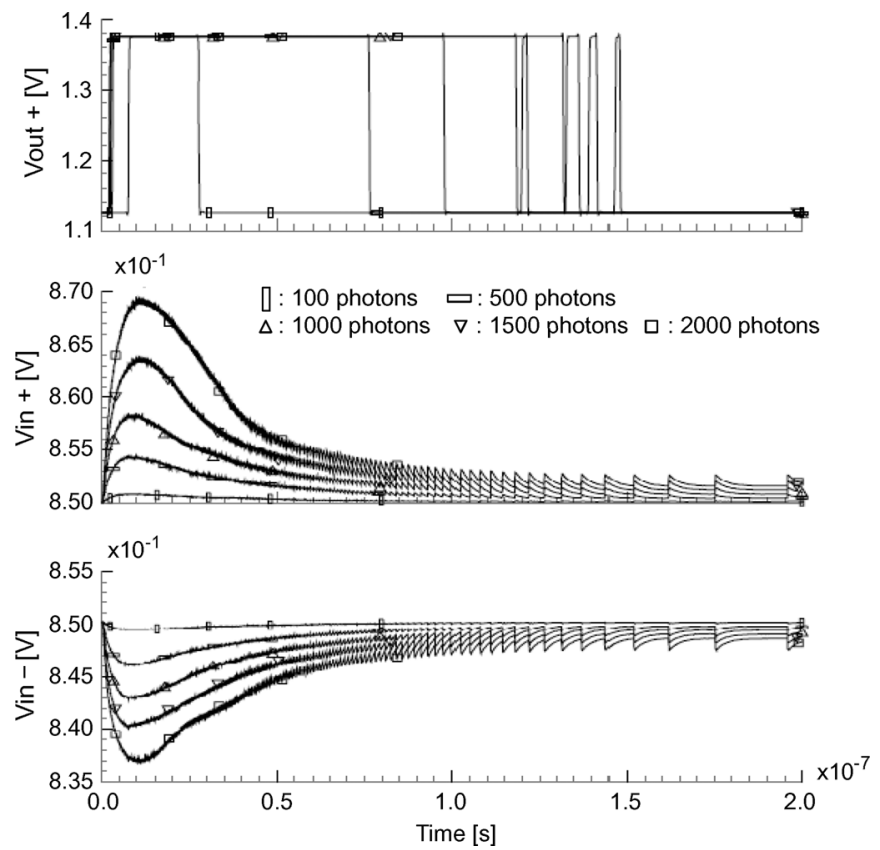

Fig. 6. SPICE simulation result of the amplifier discriminator response for a signal ranging from 100 photons to 2000 photons. Top signal is the discriminator output, the bottom two signals are the signals seen at the differential amplifier inputs. For this simulation, the SiPM HSPICE model is driven by packets of 10 photons.

TABLE III

Simulated Time WALK FOR THE First ARriving PHOtons of a LSO Signal (With 2000 Photons In Total. OVERBias OF THE SiPM IS SET TO 1.5 V.)

\begin{tabular}{|c|c|c|c|c|c|c|c|c|}
\hline \# Photon & 1 & 2 & 3 & 4 & 5 & 6 & 7 & 8 \\
\hline walk [ns] & 1.8 & 1.52 & 1.41 & 1.35 & 1.3 & 1.29 & 1.29 & 1.29 \\
\hline
\end{tabular}

signal typically consists of an average of 30 photons within the first nanosecond.

To estimate the channel sensitivity to the first photons arriving at the detector input, our Monte Carlo model (Section III-A) generated a random time sequence of photons which served as input to the SiPM-SPICE model. Simulations have been carried out from as low as one photon up to the saturation point of the amplifier input stage which is at $\approx 10$ photons. The corresponding time walk in signal response was also obtained from these simulations and is shown in Table III. This demonstrates that single photon detection is feasible with proper timing correction and, that above 6 photons, time walk is constant.

Knowing that from 6 photons onwards the time walk is constant, we have also estimated (via Monte Carlo) the LSO inherent time jitter for two cases of 10 LSO photons arriving at the detector over two different time sequences and hence producing a time shift in the discriminator response. Under these circumstances alone, already a time difference of 99 ps is observed (Fig. 7).

\section{Coincidence Measurements With ${ }^{22} \mathrm{Na}$}

\section{A. Experimental Setup}

As shown in Fig. 8, a $4 \mathrm{MBq}{ }^{22} \mathrm{Na}$ source is placed between two identical detector systems back-to-back in coincidence. Each of these consists of a SiPM Hamamatsu S10362-33-

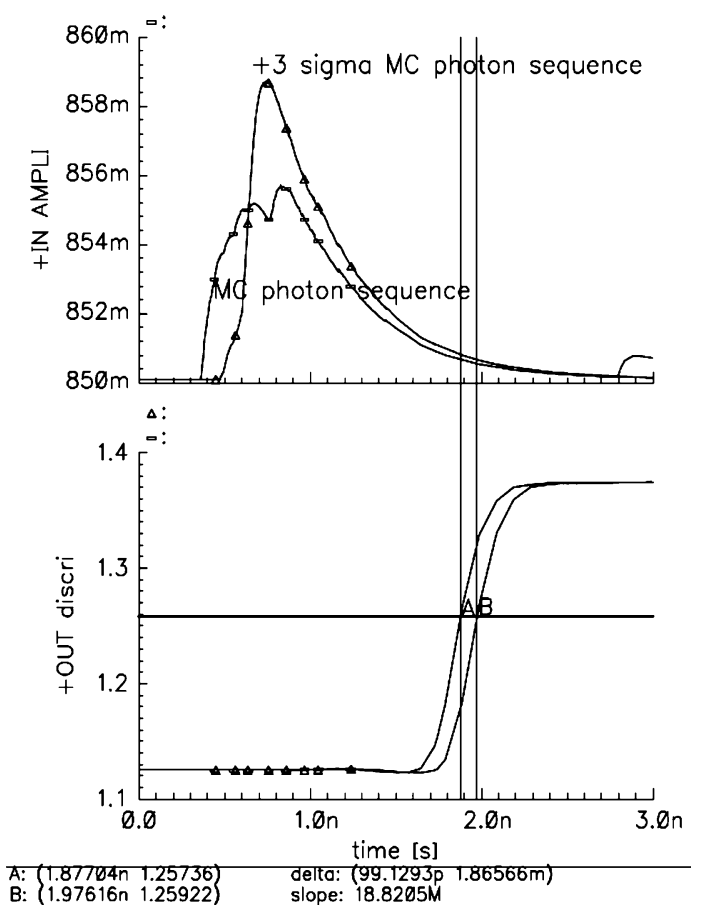

Fig. 7. Jitter simulation for two timing sequences of 10 photons. The time difference induced by photon statistics reaches 99 ps by estimation. The SiPM over-bias is $1.5 \mathrm{~V}$

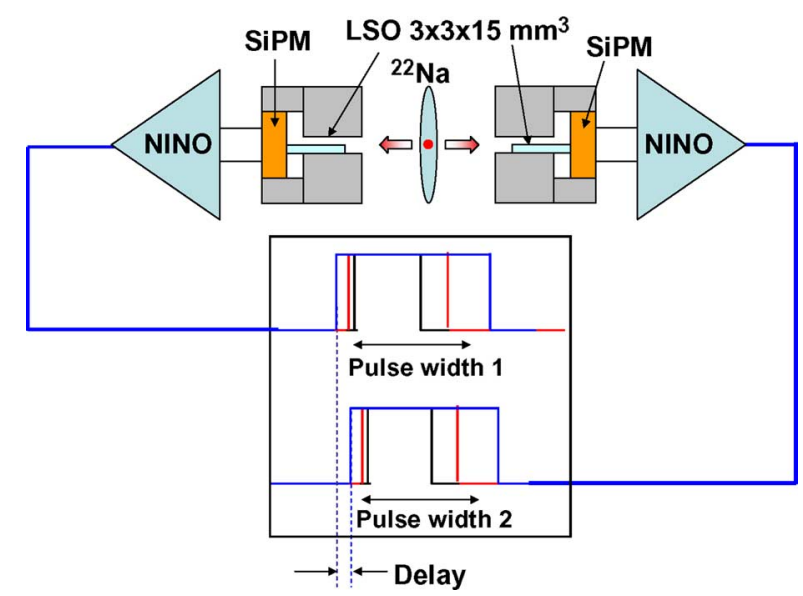

Fig. 8. Schematic of the setup

050C, connected to a LSO crystal of $3 \times 3 \times 15 \mathrm{~mm}^{3}$ size and with a light yield (photon output) of $\approx 20,000$ photons $/ \mathrm{MeV}$. The output of the SiPM is connected differentially as described before to the NINO chip. The crystals are wrapped with Teflon and coupled with silicon grease to the SiPMs. Both detector systems are at a distance of $\approx 2 \mathrm{~cm}$ from the ${ }^{22} \mathrm{Na}$ source and read out with a digital oscilloscope LeCroy WavePro 7100.

\section{B. Measurements and Results}

For each trigger three measurements were made: the output pulse width 1 and 2 of the left and right detector respectively, and the measurement of the delay between the pulses. The measurement of the pulse width gives the energy information, as shown in Fig. 9. 

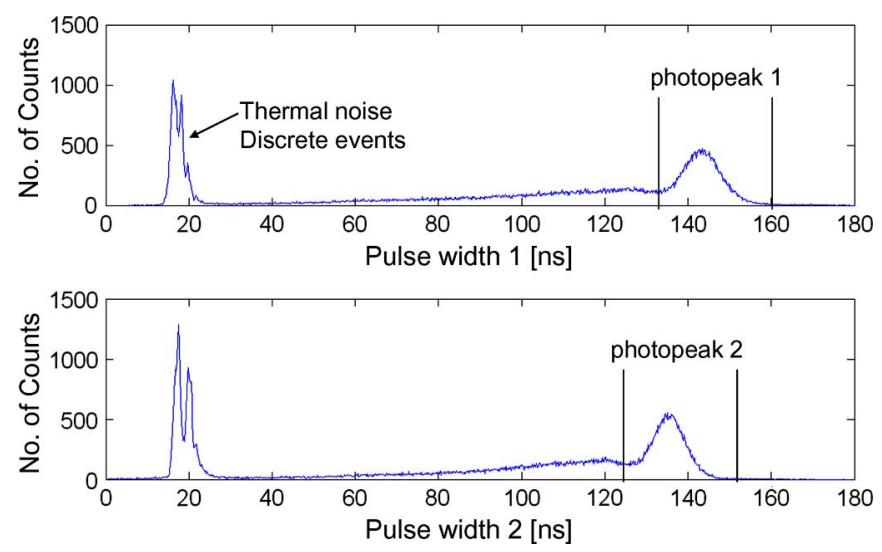

Fig. 9. Pulse width histograms of the left and right detection systems, on top and bottom, respectively. Energy resolution with this setup is $\approx 20 \%$ FWHM.

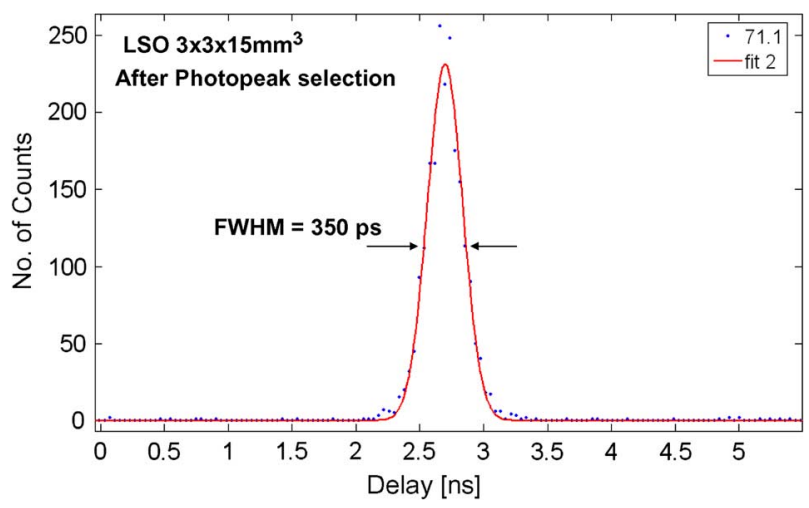

Fig. 10. Coincidence time resolution after photopeak selection.

The histograms show 3 important regions. The first one between $15 \mathrm{~ns}$ and $30 \mathrm{~ns}$ corresponding to noise events from the SiPMs. The thresholds of the NINOs are set to the level of one photon so that events as low as thermal noise (1 photon) and optical crosstalk ( 2 photons) can be observed. The second region, between $30 \mathrm{~ns}$ and $\approx 130 \mathrm{~ns}$ corresponds to Compton events, whereas the third region, between $130 \mathrm{~ns}$ and $165 \mathrm{~ns}$ covers the photopeak.

Only events in the photopeak of both detection systems are selected. The resulting delays are histogrammed in Fig. 10 which shows the coincidence time resolution of the detection system being 350 ps FWHM.

It should be noted that for photopeak events, the time walk correction improves the results by only $\approx 2 \mathrm{ps}$, which is negligible compared to the results obtained.

Reproducibility measurements have been done, changing each time the crystal wrapping and the silicon grease. From this it turns out that the coincidence time resolution varies between $326 \mathrm{ps}$ and $352 \mathrm{ps}$ FWHM. This is most likely caused by temperature effects. Improvements are made through temperature stabilization inside the dark box.

\section{INTERPRETATION AND DISCUSSION OF RESULTS}

To put the measured coincidence time resolution of $350 \mathrm{ps}$ FWHM into context with our estimates of achievable timing precision we will discuss and summarize all the parameters

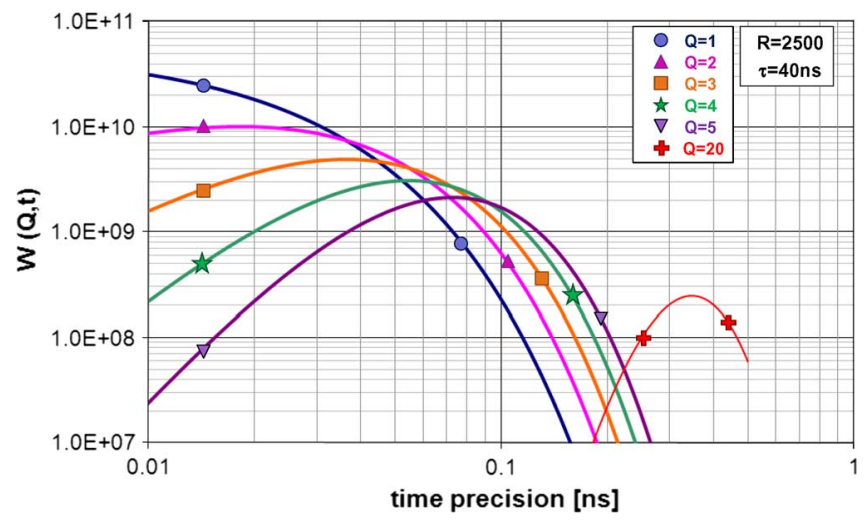

Fig. 11. Probability density of detecting between 1 to 5 and 20 photoelectrons.

influencing the overall timing precision in our experimental system. Apart from the jitter effects arising from the noise of the electronics and the photodetector, as already discussed in Section II, this section gives a comprehensive/short overview of all other factors influential to a certain degree to the detectors' final time resolution.

\section{A. Timing Limitation Due to Photostatistics}

The most prominent effect on timing resolution is limitation in photoelectron statistics. An exhaustive investigation of these effects is found in [22]-[26]. Already Section III addressed this question treating the issue with Monte Carlo simulation. In addition, on purely statistical grounds, the time resolution derives from the probability density of detecting a single photoelectron, say the $\mathrm{Q}^{\text {th }}$ p.e. out of an ensemble $\mathrm{R}$ produced within a defined time window, and the photon emission rate. Fig. 11 shows the probability density for the 1st, 2nd, 3rd, 4th, 5th and 20th photoelectron and hence their latency or timing uncertainty derived from the maximum of each curve. This maximum represents the most probable time interval in which the $\mathrm{Q}^{\text {th }}$ photon will be detected.

Recently, these estimates were refined in an attempt to include, next to the scintillation decay time (40 ns), the effect of the rise time of the scintillation process [27]. This induces an additional time lag for the scintillation photons arriving at the detector, as shown in Fig. 12 for the case of $\tau_{\text {rise }}=500$ ps. Using a LYSO crystal we have in fact investigated the scintillation rise time ourselves using a $10 \mathrm{GHz}$ scope, where a 500 ps rise time provides the best fit to the measured photodetector pulses (to be published in a separate paper). Consequently, the arrival time of the first photon is now within 200 ps FWHM and that of the second shifted to $\approx 370 \mathrm{ps}$ FWHM. It should be noted again that the above Monte Carlo simulations did not include these effects of $\tau_{\text {rise }}$ at this time.

\section{B. Geometric Effects of the Scintillator on Time Resolution}

There are actually two effects on the time resolution that are accounted to the size of the scintillator alone. The first is attributed to the variance/fluctuation of the conversion point of the incoming gamma rays anywhere inside the oppositely located crystals. The second is governed by the random travel paths of the scintillation photons also subject to multiple reflections on the crystal walls. 


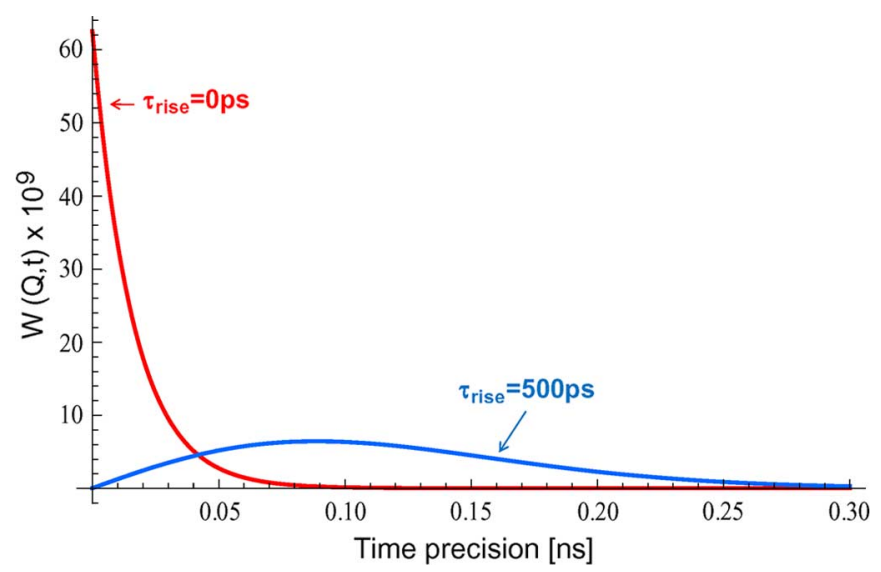

Fig. 12. Effect of scintillation rise time on time resolution. For the case of the first photon to be detected, either in a scenario of instantaneous photon emission $\left(\tau_{\text {rise }}=0 \mathrm{ps}\right)$ or one with $500 \mathrm{ps}$ rise time $\left(\tau_{\text {rise }}=500 \mathrm{ps}\right)$.

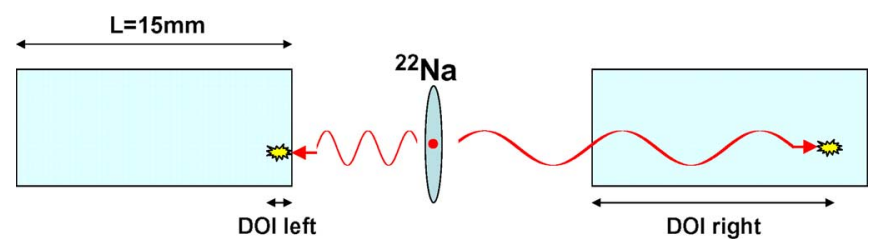

Fig. 13. Fluctuation of the $\gamma$ transit time in the scintillator before interaction.

1) Gamma Transit Time: In the extreme case, where one gamma converts at the near and the other at the far end of the other crystal (see Fig. 13), the maximum time difference $\Delta t_{\max \gamma}$, would be given by $\mathrm{L} / \mathrm{c}(=50 \mathrm{ps})$, where $\mathrm{L}$ is the length of the crystal $(15 \mathrm{~mm})$ and $\mathrm{c}$ the speed of light. Given the random nature of the conversion location we assume $\Delta t_{\max \gamma}$, to be equivalent to the $6 \sigma$ range of a Gaussian, allowing us to deduce the standard deviation of such a distribution. Hence, $\sigma_{\gamma}=1 / 6 \times \Delta t_{\max \gamma}=50 / 6=8.3$ ps or $\approx 20$ ps FWHM coincidence. Thus, the effect is small if not negligible at the level of crystal lengths below a few centimeters.

2) Scintillation Photon Transit Time: Similarly from the crystal's length and the refractive index of the crystal and the coupling medium (see Fig. 14), one can estimate the various photon travel paths and hence the corresponding time uncertainty, taking into account both direct and deflected photon trajectories inside the crystal until the light emerges from it. As described in detail in [19], [21]. the photon transit time is assumed to be of Gaussian nature, and the maximum variance confined within $6 \sigma$ of that distribution. With that approach one can show that the time uncertainty $\sigma_{\text {photon }}$ depends linearly on the crystal length (in the present case $\mathrm{L}=15 \mathrm{~mm}$ ) and the ratio of the associated indices of refraction:

$\sigma_{\text {photon }}=\frac{1}{6} \cdot \frac{2 L}{c} \cdot \frac{n_{L S O}^{3}}{n_{\text {Si grease }}^{2}} \approx 51 \mathrm{ps}$ or $\approx 120 \mathrm{ps} \mathrm{FWHM}$

This effect is sizable for crystals commonly in use for PET and should hence not be neglected. Therefore to minimize the

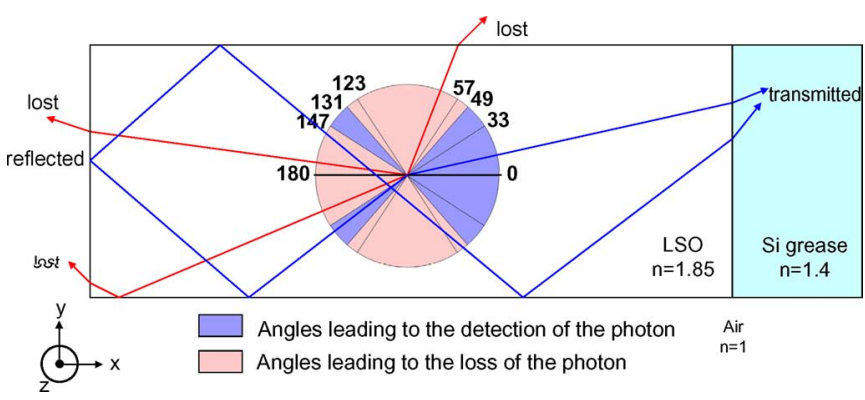

Fig. 14. Fluctuation of the scintillation photon transit path in the scintillator. Bare crystal with specular reflections.

TABLE IV

List OF CALCULATED TIMING DisPERSIONS

\begin{tabular}{|c|c|c|c|}
\hline $\begin{array}{c}\text { Front end Amp. } \\
\text { and SiPM }\end{array}$ & $\begin{array}{c}\text { Photon statistics } \\
\text { with } \tau_{\text {rise }} \text { and } 1^{\text {st }} \text { p.e. }\end{array}$ & $\begin{array}{c}\text { Gamma } \\
\text { transit }\end{array}$ & $\begin{array}{c}\text { Photon } \\
\text { transit }\end{array}$ \\
\hline$\sigma_{\text {jitter }}$ & $\sigma_{\text {stats }}$ & $\sigma_{\gamma}$ & $\sigma_{\text {photon }}$ \\
\hline 8.5 & 90 & 6 & 51 \\
\hline
\end{tabular}

geometric effects on timing resolution and the associated jitters produced by the photon transit times - and to a lesser degree by the gamma transit times-short crystals should be chosen, albeit at the expense of a rapid loss in detection efficiency with decreasing crystal size.

\section{Time Jitter of the SiPM and Front End Amplifier}

As we have mentioned in Section II the effective time jitter of the SiPM-NINO ensemble was estimated to $\approx 20$ ps FWHM taking into account the shot noise of the front end electronics and the leakage current of the SiPM with the signal peaking time at $1.5 \mathrm{~ns}$.

\section{Comparison of Simulations With Measurements}

Given the independent relationship of the above phenomena affecting the system's overall time resolution we sum the individual contributions quadratically. Each time resolution is expressed in terms of the standard deviation of a Gaussian in [ps] for a single detector arm. This is summarized in Table IV:

$$
\sigma_{\text {total }}=\sqrt{\sigma_{\text {jitter }}^{2}+\sigma_{\text {stats }}^{2}+\sigma_{\gamma}^{2}+\sigma_{\text {photon }}^{2}}=103 \mathrm{ps}
$$

To compare this value with our measured coincidence time resolution of 350 ps FWHM we multiply total with $\sqrt{2}$ and 2.35 yielding a value of $\approx 345 \pm 30 \mathrm{ps}$ FWHM. Although this calculation is in good agreement with the results obtained, further measurements are needed to validate our Monte Carlo simulations and compare them with our simplified model on time resolution in TOF-PET detectors.

\section{CONCLUSION}

The first part of this work described the modeling of a complete TOF-PET detector channel using GEANT4 and HSPICE. GEANT4 has generated sequences of scintillation photons 
taking into account the characteristics of the crystal whereas HSPICE has simulated the response of the photodetector and the readout electronics.

In the second part, our work showed that under real conditions such a detection system can provide a coincidence time resolution of 350 ps FWHM, using $3 \times 3 \times 15 \mathrm{~mm}^{3}$ LSO crystals, $3 \times 3 \mathrm{~mm}^{2}$ Hamamatsu SiPMs and the NINO chip. In the mean time, however, our Monte Carlo studies have progressed to the extent that the scintillation rise time was included. These current simulation results now show excellent agreement with the coincidence time resolution measured both in this work and in another paper to be published by the same authors [28].

Finally, we have identified the different influences on time resolution in scintillator based TOF-PET detectors. Apart from the timing constraints set by the crystal geometry alone, photoelectron statistics including scintillation rise time uncertainty are the most limiting factor to TOF-PET accuracy. Jitter produced by electronics noise and SiPM leakage current will also influence the time resolution, but are estimated to be of lower importance than the above effects.

Another important outcome of this work is an estimate of the lower limit to the timing performance of a TOF-PET system. Geometrical effects of the crystal alone impose a principal limit in time precision of $\approx 120$ ps FWHM for the crystal used.

Therefore it appears rather unlikely that with current (long) LSO scintillators, a coincidence time resolution of the order of 100 ps FWHM can be reached. A large effort in developing faster and shorter crystals with an increased photon yield, as well as photodetectors with higher quantum efficiency over present devices may ultimately achieve this goal.

\section{REFERENCES}

[1] J. S. Karp et al., "Benefit of time-of-flight in PET: Experimental and clinical results," J. Nucl. Med., vol. 49, no. 3, p. 462, 2008.

[2] C. L. Kim, G. C. Wang, and S. Dolinsky, "Multi-pixel photon counters for TOF-PET detector and its challenges," in Proc. IEEE Nuclear Science Symp. Conf. Rec., 2008.

[3] C. de La Taille et al., "SPIROC, 1857," in Proc. IEEE Nuclear Science Symp. Conf. Rec., 2007, vol. N29-5.

[4] F. Corsi et al., "ASIC development for SiPM readout pixel," in Proc. 2008 Int. Workshop, Batavia, IL, JINST.

[5] P. Jarron et al., "NINO: An ultra-fast and low-power front-end amplifier/discriminator ASIC designed for the multigap resistive plate chamber," Nucl. Instrum. Methods Phys. Res. A, vol. 533, pp. 183-187, 2004.

[6] T. C. Meyer, F. Powolny, F. Anghinolfi, E. Auffray, M. Dosanjh, H. Hillemanns, H.-F. Hoffmann, P. Jarron, J. Kaplon, M. Kronberger, P. Lecoq, D. Moraes, and J. Trummer, "A time-based front end readout system for PET \& CT," in Proc. IEEE Nuclear Science Symp. Conf. Rec., Oct. 29-Nov. 1 2006, vol. 4, pp. 2494-2498.
[7] F. Powolny, E. Auffray, H. Hillemanns, P. Jarron, P. Lecoq, T. C. Meyer, and D. Moraes, "A novel time-based readout scheme for a combined PET-CT detector using APDs," IEEE Trans. Nucl. Sci., vol. 55 , no. 5, pt. 1 , pp. $2465-2474$, Oct. 2008.

[8] F. Anghinolfi, J. Christiansen, and P. Jarron, "Readout Circuit for Use in Combined PET CT Apparatus," WO2008 040384 international patent, Nov. 2, 2006, PCT/EP2006/010524.

[9] J. Christiansen et al., "A flexible multi-channel high resolution time to digital converter ASIC," in Proc. Nuclear Science Symp. Conf. Rec., 2000, pp. 9/155-9/159, v.2.

[10] M. Despeisse, P. Jarron, F. Anghinolfi, S. Tiuraniemi, F. Osmic, P. Riedler, A. Kluge, and A. Ceccucci, "Low-power amplifier-discriminators for high time resolution detection," IEEE Trans. Nucl. Sci., vol. 56, no. 2, pp. 375-381, Apr. 2009.

[11] J. Lapington and M. Despeisse et al., "A multi-channel high-time resolution detector for high-content imaging," Nucl. Instrum. Methods Phys. Res. A, vol. 610, no. 1, pp. 123-127, 2009.

[12] E. Martin et al., "The 5 ns peaking time transimpedance front end amplifier for the silicon pixel detector in the NA62 experiment," in Proc. IEEE Nuclear Science Symp. Conf. Rec., 2009, pp. 381-388.

[13] A. Akindinov et al., "Final test of the MRPC production for the ALICE TOF detector," Nucl. Instrum. Methods Phys. Res. A, vol. 602, no. 3, pp. 709-712, May 2009.

[14] M. Kelbert, I. Sazonov, and A. G. Wright, "Exact expression for the variance of the photon emission process in scintillation counters," Nucl. Instrum. Methods Phys. Res. A, vol. 564, pp. 185-189, 2006.

[15] J. Allison et al., "Geant4 developments and applications," IEEE Trans. Nucl. Sci., vol. 53, no. 1, pp. 270-278, Feb. 2006.

[16] S. Agostinelli et al., "Geant4-A simulation toolkit," Nucl. Instrum. Methods Phys. Res. A, vol. 506, pp. 250-303, 2003.

[17] Hamamatsu Photonics, MPPC Data Sheet [Online]. Available: http:// www.hamamatsu.com

[18] S. Cova et al., "Avalanche photodiodes and quenching circuits for single-photon detection," Appl. Opt., vol. 35, no. 12, pp. 1956-1976, 1996.

[19] F. Powolny, "Characterization of time resolved photodetector systems for PET" Ph.D. dissertation, Université de Neuchâtel, Neuchâtel, Switzerland, 2009 [Online]. Available: http://doc.rero.ch/record/ $12683 ? \ln =$ de

[20] Photon Cross Sections Database [Online]. Available: http://physics. nist.gov/PhysRefData/Xcom/Text/XCOM.html

[21] S. Brunner, "New methods for improvement of time of flight positron emission tomography," M.S. thesis, CERN, Geneva, Switzerland, Mar. 2010.

[22] R. F. Post and L. I. Schiff, "Statistical limitation on the resolving time of a scintillation counter," Phys. Rev., vol. 80, p. 1113, 1950.

[23] F. J. Lynch, "Improved timing with NaI(Tl)," IEEE Trans. Nucl. Sci., vol. NS-13, no. 3, pp. 140-147, Jun. 1966.

[24] C. H. Westcott, "A study of expected loss rates in the counting of particles from pulsed sources," Proc. Royal Society, vol. A194, p. 508, 1948.

[25] A. G. Wright et al., "Exact expression for the variance of the photon emission process in scintillation counter," Nucl. Instrum. Methods Phys. Res. A, vol. 564, pp. 185-189, 2006.

[26] W. W. Moses et al., "Factors influencing timing resolution in a commercial LSO PET camera," IEEE Trans. Nucl. Sci., vol. 53, no. 1, pp. 78-85, Feb. 2006.

[27] Y. Shao, "A new timing model for calculating the intrinsic timing resolution of a scintillator detector," Phys. Med. Biol., vol. 52, pp. 1103-1117, 2007.

[28] M. Goettlich et al., "Commissioning and modeling of a multi-channel ToF-PET system with multi-pixel photon counter read-out," Nucl. Instrum. Methods Phys. Res. A, to be published. 